\title{
Integrative Clinical Ethics Support in Gender Affirmative Care: Lessons Learned
}

\author{
Laura Hartman ${ }^{1}$ (D) - Guy Widdershoven ${ }^{1}$ - Annelou de Vries ${ }^{2}$. \\ Annelijn Wensing-Kruger ${ }^{2,3} \cdot$ Martin den Heijer $^{2} \cdot$ Thomas Steensma $^{2,3}$. \\ Bert Molewijk ${ }^{1,4}$
}

Published online: 16 May 2019

(c) The Author(s) 2019

\begin{abstract}
Clinical ethics support (CES) for health care professionals and patients is increasingly seen as part of good health care. However, there is a key drawback to the way CES services are currently offered. They are often performed as isolated and one-off services whose ownership and impact are unclear. This paper describes the development of an integrative approach to CES at the Center of Expertise and Care for Gender Dysphoria (CEGD) at Amsterdam University Medical Center. We specifically aimed to integrate CES into daily work processes at the CEGD. In this paper, we describe the CES services offered there in detail and elaborate on the 16 lessons we learned from the process of developing an integrative approach to CES. These learning points can inform and inspire CES professionals, who wish to bring about greater integration of CES services into clinical practice.
\end{abstract}

Keywords Clinical ethics support · Moral case deliberation - Gender affirmative care $\cdot$ Integrative clinical ethics support

\section{Introduction}

A growing number of practitioners and experts see clinical ethics support (CES) for health care professionals and patients as part of good health care. Especially in the Netherlands, CES services are commonly divided into three main categories:

Laura Hartman

la.hartman@vumc.nl

1 Department of Medical Humanities, Amsterdam UMC, Amsterdam, The Netherlands

2 Centre of Expertise on Gender Dysphoria, Amsterdam UMC, Amsterdam, The Netherlands

3 Department of Medical Psychology, Amsterdam UMC, Amsterdam, The Netherlands

4 Faculty of Medicine, Centre for Medical Ethics, Institute of Health and Society, University of Oslo, Oslo, Norway 
(1) clinical ethics consultation, (2) clinical ethics committees, and (3) moral case deliberation (MCD), or a combination of these three (Dauwerse et al. 2014). Providing CES is a dynamic enterprise and often requires pioneering work and innovation. Not enough is known about what kind of CES works best in what context and what kind of impact and outcomes one should strive for by means of CES (Schildmann et al. 2013, 2017). Often, CES activities are performed as isolated, one-off activities whose ownership and impact remain unclear. There are known cases of clinical ethics committees writing guidelines, which are subsequently not known to the professionals and patients in their facility.

MCDs are meetings, guided by a trained facilitator, in which professionals reflect on an ethical issue they have encountered (Molewijk et al. 2008). MCDs generally take place on a ward, last 60-90 minutes and can be structured according to various conversation methods in which one concrete ethical issue is explored and discussed by the whole group. MCDs are part of a movement within CES that emphasizes the importance of bringing CES closer to actual care practices (Abma et al. 2010). The MCD facilitator engages and involves care practitioners, encouraging them to reflect on ethical issues in a dialogue. MCD makes care practitioners personally responsible for investigating and reflecting upon ethical issues, as opposed to merely taking advice or unquestioningly following a guideline or protocol (Abma et al. 2010). Although MCD is generally considered a valuable form of CES (Seekles et al. 2016; Janssens et al. 2015; Hem et al. 2015), it also faces problems related to ownership and impact:

1. MCD sessions are mainly planned and prepared by the MCD facilitator and not by the teams themselves. On some teams, MCDs frequently have to be rescheduled due to busy work schedules.

2. After MCD sessions, staff usually have to return to a busy workfloor right away. This can make MCD an isolated experience, i.e., not connected to or integrated with regular practice and work processes.

3. Due either to time constraints or a lack of policy, there is often insufficient followup by team members or MCD facilitators regarding the outcomes of the MCDs. Sometimes this leads to the situation that systemic causes of the ethical issues as signals within the MCDs are subsequently not dealt with.

4. The facilitator's relationship or contact with the department/team or ward tends to be limited to the facilitated MCD sessions. Also, MCD facilitators sometimes alternate, so it becomes difficult for the staff and the facilitator to establish and maintain a cooperative relationship. The MCD facilitator usually has limited knowledge of the team's daily work and processes, which puts a constraint on the depth of understanding that can be achieved regarding the specific ethics cases discussed in the MCD sessions.

In order to overcome these difficulties related to ownership, proximity to actual work processes, and impact/follow-up regarding systemic causes of the ethical issues, we set out to explore how CES activities could be more deeply integrated into a team's daily care processes. This paper describes the process-pursued 
openly and in cooperation with the CEGD team-of integrating MCDs into, and developing new kinds of CES activities within, the CEGD's daily work processes. Elsewhere, we describe the theoretical background of our approach to integrating CES activities into daily care processes (Hartman et al. forthcoming). Here, we describe the experiences of those involved in the process of integrating CES, and then elaborate on the learning points. These learning points and the viewpoints expressed on integrative ethics support in this paper may be informative and inspiring to CES professionals who wish to more deeply integrate clinical ethics support into clinical practice.

\section{Background}

The Centre of Expertise on Gender Dysphoria (CEGD) provides gender affirmative care for individuals who experience Gender Dysphoria (GD) in the Netherlands. GD refers to the distress resulting from an incongruence between one's gender identity and the gender assigned at birth (Gender Identity Research and Education Society (GIRES) 2006). Gender affirmative care includes feminization and masculinization through hormone therapy and/or surgery. See Box 1 for a more detailed description of the team and the type of GD care offered in the Netherlands.

Treatment teams working with transgender youth and adults often face ethical questions (Gerritse et al. 2018). These include questions such as: whether a patient's co-existing psychiatric problems are so serious that transgender treatment should be stopped or should not take place; whether or not to pursue treatment of a youngster whose parents disagree with the recommended course of treatment; whether it is necessary to reach a multidisciplinary team consensus on the whole transgender care trajectory before beginning the first phase of the treatment; and under what circumstances an exception may be made to the current treatment protocol.

Discussion of these moral dilemmas is often complicated by several factors: (a) GD is a relatively new domain and views on gender dysphoria and its treatment are the subject of continuous development and debate, both within the profession and in society at large; (b) there is only low-grade scientific evidence (or none at all) for many elements of gender affirmative treatment and its long-term consequences (Hembree et al. 2017); (c) the treatment team is multidisciplinary and different disciplines sometimes have divergent views on treatment criteria, while they are highly dependent on each other under the treatment plan in the Netherlands.

To support the CEGD in these moral dilemmas, two authors began organizing regular CES services in 2013 by offering moral case deliberations. They based their work on extensive MCD expertise gained from various research projects. Their projects have focused on MCD implementation (Weidema 2014), MCD evaluation and outcomes (Snoo-Trimp et al. 2016), and training MCD facilitators (Stolper 2016). MCD is one of four types of CES offered at our academic hospital. The others are: a clinical ethics committee, ad hoc consultancy by ethicists, and moral counseling 
for patients and families by spiritual care; all four CES services are provided for the hospital as a whole.

To resolve the problems associated with MCD as described in the introduction, we gradually adjusted and developed our clinical ethics support to suit it to the CEGD context and in accordance with our initial viewpoints on integrative CES (Dauwerse et al. 2012). During the programmatic development of integrative CES, we continuously aimed to integrate CES into the CEGD's working practices.

In our programmatic development of integrative CES, we drew inspiration from responsive evaluation research methodology (Guba and Lincoln 1989; Lincoln 2003; Abma and Widdershoven 2014). A central aspect of responsive evaluation is the involvement of stakeholders in the research process, who jointly formulate projects' aims and evaluation criteria. In this case, the stakeholders were the professionals working at the CEGD. In the future, we aim to include more stakeholders, in particular patients and their families. A second characteristic of responsive evaluation is that the design of both the evaluation process and the further development of CES emerge through an open, cyclical process. In our program, the CEGD professionals were continuously involved in both the implementation and further development of CES, in part by participating in and co-creating the evaluation of CES activities. The types of CES services offered were not planned out or decided in advance, but evolved during the process (Abma et al. 2009). We chose the term 'integrative CES' to describe our activities because it emphasizes the dynamic aspect of programmatic development. This openness enabled us to continuously respond to the changing CES needs of the CEGD team. These CES needs were jointly formulated by the CES professionals and the CEGD team.

\section{Box 1 The Work Processes of the CEGD in The Netherlands}

The local clinical guidelines of the CEGD adhere closely to internationally established standards of care (WPATH).

The local clinical guidelines make a distinction between prepubertal children and adolescents. In a series of diagnostic sessions, prepubertal children and their families receive an evaluation of the child's gender identity and co-occurring psychological and social functioning. Advice, but no medical care, is offered. Adolescents may, after careful assessment, be eligible for (fully reversible) puberty suppression. This is meant to create time for an extended diagnostic phase during the onset of pubescence (11-12-years-old) and is done only when the patient meets eligibility criteria: long-term gender dysphoria, comprehension of the medical consequences, and no psychological or social problems which could interfere with the diagnosis. Medical gender affirmative care by means of crosssex hormones, which is partially reversible, may be administered to patients 15-16 years of age and by medical prescription only. Medical gender affirmative care by means of surgical treatment is provided only to adults (18+); all postoperative transgender individuals who receive such treatment require lifelong continuation of cross-sex hormones. 
Clinical guidelines for adults are similar to children and adolescents. First, there is a careful diagnostic phase in which a mental health care professional aims to determine the following: whether GD is present and its severity; potential co-morbidities; the stability of the social support system; the coping mechanisms; whether the patient comprehends GD and the long-term consequences of medical care; and the ability to comply with therapy and medically necessary follow-up appointments.

Within the medical gender affirmative care trajectory, decisions regarding eligibility for a given phase are based on multidisciplinary consensus in team meetings and take the individual patient's wishes into account.

In the Netherlands, care for transgender persons takes place at specialized Centers of the University Medical Hospitals of Amsterdam and Groningen. When the current study was conducted, there was also a third center in Leiden, but it is now closed. Dutch transgender care requires close multidisciplinary cooperation between the two care centers and between psychologists, psychiatrists, endocrinologists, plastic surgeons, urologists, pediatricians, nurse specialists, and gynecologists.

\section{Description of CES Services}

In the following section (subheadings "Moral Case Deliberation at CEGD Policy Meetings" through "Ethics Logbook"), we describe the process of developing and offering various CES services. We had no pre-existing blueprint of what integrative CES entailed; we gradually developed our services in line with the team's CES needs and our evolving insights on CES. For each service we describe in this section, we will list the lessons learned that are applicable to the development of integrative CES. See Fig. 1 for an overview and timetable of all the integrative CES activities.

\section{Moral Case Deliberation at CEGD Policy Meetings}

Our first CES service came in response to a request for moral case deliberation during the policy meetings and vision meetings of the CEGD clinical teams for adult and youth care in 2013 (so a total of four meetings). At the annual policy meetings, about 40 professionals from the team for adult care and 20 from the team for youngsters, of all involved disciplines (i.e., psychologists, plastic surgeons, gynecologists, urologists, endocrinologists, psychiatrists, pediatricians and nurse specialists), meet for one full day or afternoon and discuss the latest developments related to the care they provide.

In 2013 and 2014, a colleague of two of the authors gave an introduction to MCD (expectations, goals, expected attitude during the MCD), followed by parallel MCD sessions structured according to the dilemma method (Stolper et al. 2016). There were about 12 participants per MCD, so the number of parallel MCDs organized depended on the number of attendees. The various professional disciplines involved in gender affirmative care were divided equally among the MCDs to encourage interdisciplinary exchange. In each MCD, one professional brought forward a case. The only precondition for this case was that the case presenter had to have experienced a 


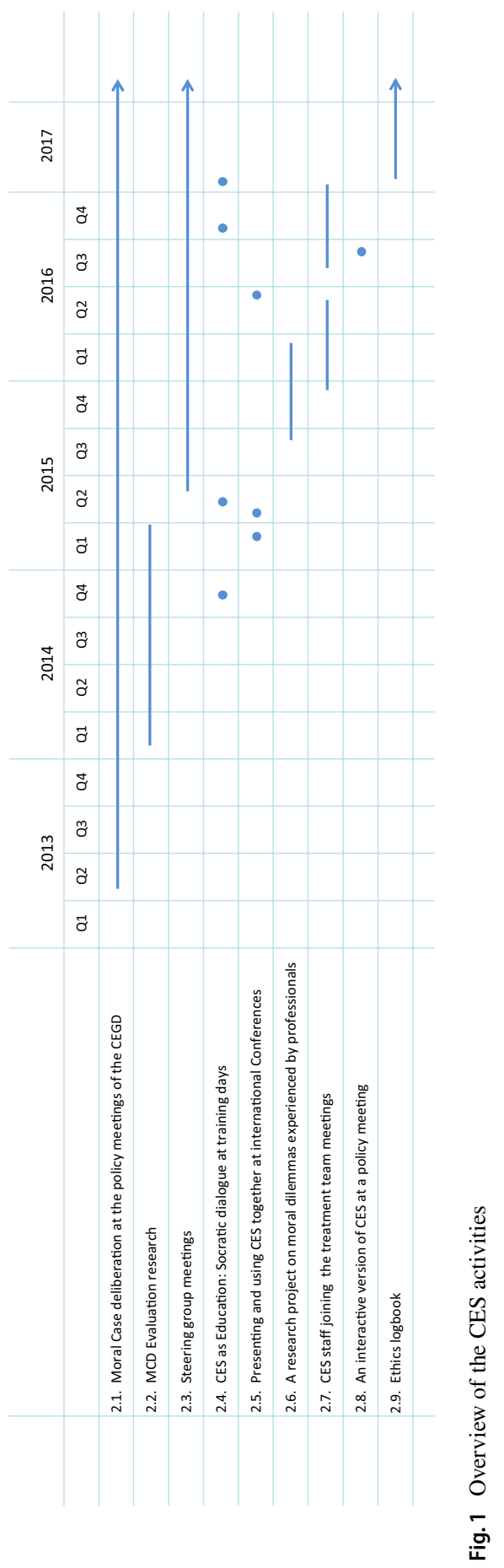


moral dilemma. The trained facilitator (Stolper et al. 2015) guided the dialogue and drew up a report on the meeting, which was checked by the case presenter and then sent back to each participant.

Between 2013 and 2016, we offered 20 facilitated MCDs at 13 CEGD policy meetings; the policy meetings proved to be an ideal setting for MCDs because every discipline involved in GD care was present and there were no emergencies, pagers going off or other care-related responsibilities disrupting the deliberations.

We worked with a dedicated team of liaisons, consisting of CEGD members and staff from the Department of Medical Humanities of Amsterdam UMC. Continuity during the policy meetings was ensured by the presence of at least one of the liaisons from the authors. One author had the lead coordinating role, and another author played a back-up supervising role. Working with a dedicated, unchanging team of liaisons (from both the CES staff and the team that "receives" CES) proved to have several advantages:

1. The liaisons provided continuity regarding the practical and organizational details and previous arrangements;

2. The CES providers gradually became familiar with the specific type of care the team provided;

3. A steady duo provided a recognizable point of contact for questions, etc.;

4. This setup contributed to continuity between the different MCDs with respect to both the content of the cases and the way the MCD processes evolved.

During this period, two authors were also present at the CEGD ward for one morning and observed a number of consultations in order to familiarize themselves with the type of care provided there and to establish informal contact with the CEGD professionals outside the MCD context. This not only gave these two authors a better feel for the type of care provided, but also for more practical aspects of the care such as the type of rooms in which the consultations take place, the setup of the waiting room area, etc. Thanks to their observations, these two authors were also able to point out possible ethical issues that had not been identified as such by CEGD professionals.

Lesson 1. MCDs are beneficial when the team is given time to reflect and there is time to address the relationship between the issues discussed during MCDs and policy.

Lesson 2. A dedicated pair of CES facilitators ensures continuity and a regular point of contact.

Lesson 3. Being present at the ward and observing consultations allows CES staff to familiarize themselves with the type of care provided and to establish informal contact with the team that receives CES.

\section{Evaluation Research}

In the context of a grant for empirical-ethical research on moral controversies in gender treatment, two authors decided to evaluate the MCDs provided at the CEGD policy meetings. The evaluation study was collaboratively conducted by the 
Department of Medical Humanities at Amsterdam UMC and the CEGD Amsterdam-Leiden gender teams. ${ }^{1}$ The two authors collected the empirical data and analyzed the data. A student took part in the research as an intern. ${ }^{2}$ Because of limited space we can only briefly summarize the outcomes of this evaluation study. The full results of the study will be published in a paper co-authored by staff from the CEDG and the Department of Medical Humanities (Vrouwenaets, forthcoming).

The evaluation study's results showed that the MCDs at policy meetings had several beneficial effects, but also left room for improvement (Vrouwenaets, forthcoming). Several interviewees expressed appreciation for the fact that all MCD participants were encouraged to contribute and that disagreements were discussed more constructively and less polemically than they were accustomed to. The dilemma method's structure ensures that participants take the time to listen to one another rather than reinforcing their wish to persuade others of their own point of view. This enabled all participants in the MCDs to formulate the relevant arguments. Furthermore, the dilemma method encourages participants to incorporate the merits of, and arguments for, "the other side" of the dilemma into their own viewpoint, instead of stimulating them to defend or become entrenched in their own positions and discredit the opinions of colleagues.

Two authors experienced several problems during the evaluation study process. It proved difficult to persuade staff at the CEGD to make time in their work schedules for the evaluation. Additionally, some CEGD professionals had the impression that their participation in this process was solely for the CES staff's benefit, to evaluate "their" CES activities. To address this issue, the two authors openly discussed and explored possible aims and ownership of the evaluation study. They emphasized that the evaluation research was not an end in itself, but a necessary means to develop an integrative CES approach in that it would enable them to adjust CES to the staff's needs and to increase the CES's usefulness and impact (Weidema et al. 2016). Hence, a guiding principle in the evaluation study was that it was not merely an evaluation of the CES as a product, but a means of jointly learning from the staff's CES experience in order to better address their needs and challenges (i.e., a responsive evaluation study). In the researchers' view, the specific aim of the evaluation study is not merely to gather data for its own sake, but to advance the collective process of implementing and improving the usefulnees of CES. Given this guiding principle, it became all the more important to follow up on the CES evaluation results. See 2 and 3.

The evaluation study process gave the two authors an opportunity to establish contact with the team in a new way (as compared to the kind of contact made when facilitating of MCD sessions). This enabled them to reveal and discuss information that would not have been available to them otherwise. This information, in one

\footnotetext{
1 We were able to perform this evaluation study as part of a larger research project that was coincidentally being conducted in the Leiden team: "Controversies surrounding suppression in adolescents with gender identity disorder: moving forward the ethical debate and creating an international guideline". For more information about the results of this project see, ZonMw (2019).

2 In total, six MCDs, six semi-structured interviews, the EURO-MCD questionnaire (T0 $(n=34)$ and T1 $(\mathrm{n}=22))($ Svantesson et al. 2014), and two multidisciplinary focus groups were included as data.
} 
instance, related to the professionals' experiences during a particular MCD, and to information about the histories of, and follow-ups on, some of the moral themes discussed during the MCDs. Another advantage of the evaluation study process was that it allowed for more informal reflection on the cooperation between CES staff and clinical staff. Furthermore, the evaluation research encouraged the CEGD staff to actively think about the kind of CES they preferred and needed; it made them aware of their own role and responsibility in developing CES. This made them more of a co-owner, rather than merely a receiver, of the services provided to them. We also received information on the criticisms expressed about MCDs. Under the next subheading, we will describe how we addressed these critical remarks. Overall, we believe that the information produced proves the value of investing time in evaluating CES services in addition to the basic work of organizing CES services and dialogues on moral cases.

Lesson 4. Combining CES activities with a responsive evaluation study delivers important insights into the usefulness of CES, improves the implementation process, and creates a shared sense of ownership of and responsibility for the use of CES in clinical practice.

\section{Steering Group Meetings}

Two authors decided to contact a number of key individuals at the CEGD with whom they could reflect on the outcomes of the evaluation study and plan concrete steps to improve the CES services offered. They established a small CES steering group composed of the following authors of this contribution: LH, BM, AV, AW, TS and $\mathrm{MH}$. The steering group members were also part of the CEGD management team and as such played a formal role at policy-making level.

The first criticism some professionals expressed was that they felt the pace of MCD was too slow. However, a slow pace is a crucial part of MCD. Part of the Socratic attitude is to slow down the thinking process, to listen to one another, and to make room for diverging viewpoints (Kessels 2009). That this is experienced by some rather action- and solution-oriented professionals as burdensome or frustrating, does not exclude the possibility that a slow MCD process can be valuable and effective. It was valuable to be made aware of this criticism from the CEGD teams because it prompted us to clarify why we think a slow MCD process is needed. For this reason, the steering group decided not to reduce the amount of time devoted to MCDs during policy meetings.

The second criticism we received was that similar cases were presented in several different MCD sessions. The chance that this will occur is inherent to the MCD method in that it is the team members themselves who select their own moral issues to discuss. Furthermore, each case can produce new insights, no matter how closely it resembles a previous one. Besides, if the case presenter still experiences a given case as a moral dilemma, then it is evident that the relevant moral theme has not yet been adequately dealt with to satisfy all team members. At least part of the team is still grappling with the issue, in other words. Nevertheless, this criticism made it clear that we needed to explicitly deal with how the theme could be better addressed and to enumerate what had already been learned from the previous case(s) and what 
still needed to be explored. The MCDs did make the team aware of moral issues that continued to resurface and were not being dealt with to everyone's satisfaction. This provided an argument for supporting the team by also addressing these issues at a clinical management policy level (see "CES as Education: Socratic Dialogue on Training Days").

The third critical theme from the evaluation study was that there was "insufficient follow-up". Some professionals felt that important insights and action strategies developed during the MCDs had not resulted in the necessary action. For instance, the participants in one MCD concluded that it was unclear which forms needed to be signed regarding parental consent at the administration office. The participants concluded that it was important to investigate this and provide some clarity. However, this was not followed up because, for one thing, it was not clear who was responsible for this. To address this issue, MCD facilitators and team members started to draw up a list of issues for follow-up at the end of each MCD. They devoted more explicit attention to what was called the "harvest" of each MCD. MCD facilitators did not feel they themselves were responsible for the follow-up, but they became more active and asked the participants which insights/remarks/outcomes of the MCD they felt should be taken up and who they thought were responsible for this.

After dealing with all three criticisms, we set up regular meetings with the small steering group to discuss these points and find ways to follow up on the insights generated by the MCDs. The results of the follow-up were shared with the team at the next policy meeting. They concerned a wide variety of issues. For instance, during one MCD it was concluded that the team lacked information and expertise on religious doubts about gender affirmative care in Islam. The steering group decided that a spiritual advisor with expertise on this topic would be invited to the next policy meeting to give a lecture.

Thanks to these meetings with the steering group, the MCDs and the associated insights became more embedded within the CEGD's workflow by means of joint responsibility and greater emphasis on following up.

Lesson 5. Creating a steering group in which CES staff and management cooperate contributes to the ownership and follow-up of both MCDs and MCD evaluation results.

Lesson 6. Not all evaluative remarks made during an evaluation study should necessarily be implemented.

Lesson 7. Facilitating the team in devoting more attention to the follow-up and practical implications of MCDs contributes to more relevant and useful MCDs.

\section{CES as Education: Socratic Dialogue on Training Days}

The benefits experienced from MCD in clinical settings led the CEGD to ask whether MCD could also be used in educational contexts. In response, we began organizing MCDs during national training sessions for (mental) health specialists seeking certification to authorize legal gender changes. Due to a change in Dutch law in 2014, transgender persons can change their legal gender without satisfying the former requirements of having had sterilizing surgery or an official (psychiatric) 
diagnosis of gender dysphoria. Instead of these old requirements, they now need a signed declaration of "mental competence" by a certified expert. However, this new policy also entails moral questions, for instance: "What is sufficient mental competence?" and "Should we avoid inconsistency between experts?" To provide support, we facilitated Socratic dialogues during training sessions for health specialists (physicians and psychologists) organized by the CEGD.

A Socratic dialogue is a specific conversation method which can be used to structure an MCD. Instead of a moral dilemma (which is used in the dilemma method), it takes a conceptual question as its starting point. In this case: "What is mental competence?" We chose this method because the candidate experts did not have practical experience in assessing mental competence in this new role. The Socratic dialogues were evaluated very positively and were experienced as an opportunity for trainees to actively challenge the experts to formulate, scrutinize, and discuss their own (moral) presuppositions on mental competence. Engaging the experts in active reflection was felt to be didactically more effective than merely being on the receiving end of passive knowledge transfer, by, for instance, a lecture on mental competence. $^{3}$

Lesson 8. MCD can be used as an active, participatory component of a training session, educational program or course.

Lesson 9. The method used to structure the MCD should be suited to the setting and goals of the participants.

\section{Presenting and Using CES Together at International Conferences}

Given the rich and complex content revealed by the MCD sessions and the positive evaluation MCD received, the CEGD felt that this kind of CES could be relevant to an international audience as well. Therefore, based on our joint cooperative process, we developed an interactive workshop on ethical issues in gender affirmative care. At three international conferences specifically related to gender affirmative care, we gave presentations on both the content and the developmental process of the co-created CES. ${ }^{4}$ As mentioned in the introduction, the international field of transgender

\footnotetext{
${ }^{3}$ Also, one author facilitated an MCD for medical students in the third year of their bachelor's program doing an elective minor in "Transgender Medicine", which is coordinated by the CEGD within the VUmc curriculum for medicine students. Finally, another author presented the developed CES services during a CEGD-hosted informative event open to anyone in the public who was interested in gender affirmative care. For all these events, the author and the CEGD worked together in education and became teaching and/or lecturing colleagues, in a sense.

${ }^{4}$ The first conference was the Biennial Meeting of the European Professional Association for Transgender Health (EPATH) held in March 2015 in Ghent, Belgium. During this session, we simulated an MCD in which we asked the attendants of the session to formulate their own moral judgment on a concrete (anonymized) moral dilemma and stimulated reflection and discussion on this case. Subsequently, we presented more information about MCD and the results of the evaluation study (see "MCD Evaluation Research"). The second conference was the yearly meeting of the Netherlands/Dutch Association for Psychiatry in the Netherlands (NWP). We used the same format, but did the simulation in Dutch, at this national conference for psychiatrists. The third one, following the same format, was the biennial meeting
} 
care is active and continuously developing. As a consequence, a lively and sometimes polarized debate is now taking place, dealing with issues such as the preferred patient-physician relationship (What is an appropriate form of shared decision-making?) and the right way to deal with children who experience transgender feelings.

The CEGD felt that the MCD framework could bring about a more constructive and less polarized discussion of the moral issues at stake. AW felt it was an advantage that LH and BM were seen as outsiders and therefore neutral in these debates. A disadvantage was that $\mathrm{LH}$ and BM were less informed about the latest vocabulary and therefore sometimes used inappropriate language (Bouman et al. 2017). We experienced the collaboration at conferences as both positive and informative. LH and BM became aware of international perspectives and debates and gained a better understanding of the CEGD's position in these debates. In addition, the joint performance of presenting and executing CES at (inter)national conferences contributed to a strong team spirit, making us more than just ethics consultants who visit the ward but have no deeper connection.

Rather than telling a polished success story at the three conferences, the CEGD showed their moral doubts in an international context. They also highlighted "ethical issues" for transgender care. The attendees of the preconference stream expressed appreciation for a moral perspective on certain issues and lauded the fact that CES offers a normative framework that incorporates both a range of professional understandings and patient understandings of good care. Some attendants asked for referrals to CES professionals in their respective countries. The variety of opinions expressed did not lead to polarized discussion, but resulted in greater openness and understanding of each other's viewpoints. Unexpectedly, representatives from Dutch transgender interest associations asked both CEGD and the Department of Medical Humanities to consider closer cooperation because they felt the two organizations could be part of an equal and open dialogue. This raised a moral question; should they (or the Department of Medical Humanities) take the lead in intensifying the relationships between the professionals in gender care and patient groups, or should they leave that to CEGD?

Lesson 10. Presenting joint experiences at (inter)national gatherings and devoting attention to both content and the development and implementation of CES contributes to a sense of joint responsibility and elicits additional reflections from other cultures and/or countries.

\section{A Research Project on Moral Dilemmas Experienced by Professionals}

To create an overview and categorization of the types of moral dilemmas experienced at the CEGD, the Department of Medical Humanities and the CEGD set up

Footnote 4 (continued)

of the World Professional Association for Transgender Health (WPATH) held in June 2016 in Amsterdam, the Netherlands. Since the CEGD was host of the biennial that year, they invited LH, BM, and GW to lend support with both the pre-stream days and in a plenary session on 'ethical issues in transgender care'. At the time, the WPATH in Amsterdam was the biggest conference held to date, with about 800 attendees. 
a new research project. One of the underlying reasons for this was that the steering group felt there were many topics within the material (e.g., the reports of the MCD sessions) that required more attention than the Department of Medical Humanities could deliver due to constraints on their time and resources. Another reason was that the steering group felt that we should pay closer attention to the places where moral issues arise during the day-to-day work situation. Until that point, we had mainly focused on moral issues arising during MCD sessions and during policy and educational gatherings.

Therefore a nine-month internship was created for a master's student of medical sciences. As part of the data collection task, the student was asked to observe the CEGD's multidisciplinary meetings and ten consultations with transgender persons (including observational notes). The student also re-analyzed the reports on the MCDs held at policy meetings, and the transcribed MCDs, held interviews and focus groups from the evaluation study of MCD. Two authors and the student did most of the analysis. They had a meeting with AW to discuss preliminary findings and incorporated her comments.

We presented the preliminary outcomes to the CEGD team at a policy meeting, raising awareness of the variety of moral dilemmas the team faces and moral decisions it makes on a daily basis. This promoted the team's awareness of the inherent moral dimension of transgender care. Until then, the team didn't recognize the ethical component of their decisions and therefore neglected to explicitly discuss the underlying normative perspectives. The results of this study will be published in a paper co-authored by CEDG and the Department of Medical Humanities staff (Gerritse et al. 2018).

Although this presentation on daily moral issues was informative, it was unclear how it should be followed up. One reason for this was the sheer multitude of moral issues at stake, leaving it unclear where to begin. The steering group asked the Department of Medical Humanities to focus on two moral themes (see "An Interactive Version of CES at a Policy Meeting" and "Ethics Logbook").

Lesson 11. A presentation of the results of observed moral issues can be thought-provoking and can stimulate reflection on moral issues that the team was not aware of.

Lesson 12. Reporting observed moral issues, both explicit and implicit, requires a specific aim and structure so team members can use and process the information.

\section{CES Staff Joining the Treatment Team Meetings}

In order to integrate CES more deeply into daily care processes, the steering group decided to conduct two six-month pilots (starting at the end of 2015, and again in June 2016), in which the CES staff were to participate in during the team's weekly multidisciplinary meetings. The CES staff received additional financial compensation from the CEGD for this new CES activity. In this way, CES became even more integrated into the financial and structural arrangements of CEGD care. $\mathrm{LH}$ and BM were asked to lend support on the moral dilemmas 
and questions arising in the multidisciplinary meetings and to accentuate the moral dimensions of certain decisions or questions. A draft document was drawn up to define the roles and tasks of the CES staff, including the kinds of interactions and interventions that were to take place as well as the aims of these interventions. According to the plans, there was to be an evaluation after six months.

Since we wanted to know how useful the interventions were, we began exploring and documenting several kinds of interventions. We distinguished several categories, including:

- asking different types of questions about difficult clinical cases discussed by the team;

- asking questions aimed at clarifying the intrepretation of rules and facts;

- asking questions about underlying (but implicit) viewpoints;

- reasoning or use of arguments;

- asking questions about the communication process and the intentions behind the communication.

LH and BM also took additional observational notes about the kinds of explicit and implicit moral issues that were discussed or were not discussed. LH and BM also tried to actively point out the links between the content of the MCDs and the actual decision making during team meetings.

The CEGD steering group's request was a new CES activity for LH and BM. They questioned, reflected on, and often discussed the "right" manifestation of this new role. The steering group also explained and discussed the role, goals, and expectations amongst themselves in face-to-face and email communications. In addition, $\mathrm{LH}$ and $\mathrm{BM}$ organized a peer meeting with several experienced CES professionals from the Department of Medical Humanities, with whom they could reflect upon their new role when present at multidisciplinary team meetings.

LH and BM experienced several challenges in this new role. Firstly, it was difficult to find the time during these meetings to intervene in the discussion and encourage reflection on the normative presuppositions underlying a certain discussion or treatment plan. The meetings are held under time pressure, with less than ten minutes dedicated to each patient, and are oriented towards quick decision making. Any reflection at all soon causes a delay. Secondly, LH and BM felt that their reasoning, goals, and methods should be made known to everyone at the meeting. Since these meetings had a large number of flexible attendees, it proved a challenge to keep everyone informed about the CES staff's role. AW (the chair) experienced LH and BM 's attendance as supportive, as they more openly questioned colleagues' viewpoints and had less presuppositions.

Lesson 13. Having CES staff joining team meetings can be a useful method to integrate CES more fully into daily care processes.

Lesson 14. It is good to dare trying new CES methods, but it is essential to evaluate their contribution critically and honestly and to involve peer CES staff when reflecting on your own role. 


\section{An Interactive Version of CES at a Policy Meeting}

As described above, LH and BM's role at a policy meeting was usually to facilitate an MCD or to report the findings of the evaluation study. Classical presentation methods put those receiving and processing the information in quite a passive role, akin to that of a student who attends a lecture. To avoid this, we discussed within the steering group alternatives to simply enumerating a large number of moral topics using a PowerPoint presentation. We chose to repeat the format developed for the pre-stream done before the WPATH. The moral topic we chose was shared decisionmaking (SDM), since this theme is pervasive in many of the moral dilemmas in CEGD practice. We presented normative statements on the moral responsibilities within SDM and the different kinds of SDM ideals we encountered in our analyses of the MCD reports. Using free voting software (i.e., Socrative, which was developed for educational purposes) we asked the staff for their normative position on both the actual and the ideal situation at the CEGD vis-à-vis SDM (both for adults and children). The opinions and the values underlying them were directly and anonymously projected onto a screen and discussed with the team. This created an engaging and interactive session, which revealed the underlying values held by team members and the distribution of their normative positions with respect to SDM.

\footnotetext{
Lesson 15. Creative means of interacting and doing normative reflection (such as voting software) can be useful ways of encouraging collective reflection upon moral issues by the team and can provide staff with immediate insight and feedback on how they think with regard to a moral issue (in this case "shared decision making").
}

\section{Ethics Logbook}

Finally, from 2017 onwards, the steering group developed another new CES activity; namely, the keeping of an "ethics logbook" on a specific moral theme. In order to create focus amidst a large number of moral issues and to guarantee a stronger harvest for clinical practice and policy, one author and an additional CES staff member asked the professionals at a policy meeting which moral themes they felt needed additional focus, and what kind of moral issues they experienced regarding these specific themes. Based on the resulting enumeration by the CEGD team, the steering group concluded that it would be beneficial to zoom in on two moral themes in particular: "fertility" and "lifestyle". We decided to keep a log and to monitor the occurrence, actual discussion on, and handling of these moral issuesthemes on the ward. In order to create shared ownership of this CES activity and observe various moments, both CES staff and CEDG members started to log and monitor all events related to these two moral themes and the way they were addressed.

The reason "fertility" and "life style" (which are related to issues like smoking and BMI, for instance) were chosen for the ethical logbook is that these were themes the team often struggled with. One author and an additional CES staff member collaborated with two loggers from the CEGD on each moral theme to record in an ethics logbook all moral dilemmas, questions, and instances encountered regarding these 
two themes. This included, but was not limited to: concrete clinical cases, moral questions, arguments for and against, decisions and actions in particular cases, and differences of opinion within the team. The CES staff subsequently planned to combine the overview with relevant arguments from the bioethical scientific literature and literature on gender, and to organize an interactive meeting to provide an overall analysis of a specific moral theme that keeps rearing its head. Our aim in doing this was to make explicit the normative presuppositions and implicit ethical and moral arguments and to reveal these to the team. This would enable the CEGD and CES team to determine what action to take based on the overall analysis (e.g., draw up new guidelines/protocols or adjust existing ones, plan educational activities on a theme, etc.).

The steering group continuously discussed and evaluated the details and process of this new CES activity.

Lesson 16. Having CES and clinical staff jointly keep an ethics logbook on specific moral themes is a good way to foster shared ownership of CES and to emphasize specific themes that commonly recur in daily care practice.

\section{Discussion}

Instead of only offering isolated MCD meetings occasionally in which the MCD facilitator facilitates the MCD and after that leaves the ward again, we gradually developed new CES services in close cooperation with the CEGD clinical team and MT. We continuously strove to integrate CES into the CEGD's care practices. Responsive evaluation research, a trusting relationship, and the CES staff's resolve to integrate CES more fully into clinical practice proved instrumental to collective learning and tailoring of CES services to the team's actual needs. These needs are not just the needs that are explicitly formulated by the CEGD team, they can also be needs of the CEGD according to the CES staff.

In this paper, we have formulated 16 lessons learned in the process of jointly developing new CES activities. Some lessons correspond to insights from the literature on CES. The importance of following up on CES services, for instance, has been emphasized before (Finder and Bliton 2011; Stella Reiter-Theil 2016). Likewise, we signaled a discrepancy between the way clinical ethics consultations are often presented in clinical ethics literature (i.e., with clear beginnings and ends) and the actual experience in practice of clinical ethics consultations, which are often marked by a far more nebulous ending in which there are still several challenges to be dealt with (Finder and Bliton 2011). Moreover, the need to experiment and innovate CES, and the readiness to modify regular CES activities has been emphasized before (Stella Reiter-Theil 2016).

As cooperation between CES and CEGD staff developed, they gradually acquired a shared sense of ownership and responsibility for following up. This was promoted, among other ways, by creating the steering group, through the responsive use of the evaluation research, and by having regular CEGD team members act as loggers monitoring a specific theme for the ethics logbook. Who takes ownership and plays a leading role in 
these various activities could well change depending on the specific topic at hand, which phase the integrative CES finds itself in, and the expertise required for a specific task.

One unresolved issue is precisely how the CES staff should intervene in and contribute to MDO sessions. First of all, what kind of expertise and competence is needed for CES to contribute to MDO meetings? Secondly, what kind of interventions are appropriate? What is the normative status of the various interventions? For example, there is a difference between asking for clarification (e.g., "What is the exact policy here? What is the concrete rationale of this policy? Is this consistent with how you dealt with patient X last week?") and criticizing a specific argumentation (e.g., "Is it morally justified to not inform the patient?"). The appropriateness of specific interventions is determined by one's general point of view on ethics expertise and the aims of CES (Reiter-Theil 2009; Pedersen et al. 2010; Reiter-Theil 2016). Rather than resolving these issues on our own, we made a crucial decision to discuss the possible interventions with others beforehand and to continuously evaluate them during the process. Interventions were often discussed and evaluated with both the steering group and the CEGD staff members. We also planned a specific meeting with CES colleagues to discuss different viewpoints on various interventions, criteria for their appropriateness, and their normative status.

There is a sensitive balance between, on the one hand, being critical and explicitly normative and, on the other hand, maintaining the relationship and trust that the clinical staff put in ethicists as critical observers (Abma et al. 2010; Widdershoven et al. 2009; Simpson 2012). While being independent and critical is regarded as a crucial part of being a CES staff member, it is important that criticism is accepted and responded to. Criticism is counterproductive if clinical staff feel the need to defend themselves, or worse, consider excluding CES staff from MDO sessions. Criticism can only be constructive and effective within a mutually respectful relationship. In order to maintain such a relationship, it is necessary to jointly reflect on the way critical remarks are received, and on the aim of such remarks. Aside from ethics knowledge and analytical skills, this requires tact and relational competencies.

The integrative CES approach described in this paper bears similarities to, but also differences from, the integrated CES approach developed by the National Center for Ethics in Health Care of the U.S. Department of Veterans Affairs (Fox et al. 2010; Doran et al. 2016). Both approaches aim to integrate CES into care work processes in response to the shortcomings of traditional CES approaches. In integrated CES, however, the services provided are mainly based on one type of ethics support, i.e., the CASE approach for ethics consultants. Furthermore, integrated ethics support mainly follows a standardized approach in which pre-defined programs, structures and tasks are recommended, whereas a key characteristic of integrative CES as described in this paper is an emerging design. The word 'emerging' refers both to the kind of ethics support offered and to the means of organizing and implementing that support. We chose the adjective integrative CES to emphasize the continuous effort it requires to integrate and adjust CES services to the recipient's needs and to the needs of a health care organization. The CES services we developed and the lessons we learned raise additional theoretical questions. Why is it important to strive for integration of CES? What are the core characteristics of an integrative approach to CES? Additional research is also needed to determine the outcomes of 
an integrative approach to CES. Does it reduce moral distress in team members, for instance? We will reflect on these and more theoretical questions in another paper (Hartman et al. forthcoming).

\section{Conclusion}

This paper described the ongoing learning process of integrating both regular and innovative CES services into the CEGD's daily work processes. In this process, CES and CEGD staff functioned as collective owners and developers of the CES services offered. In general, MCD and other CES services are often isolated ethics activities that do not trickle down to the actual care practices on the workfloor. However, the participants in MCDs and other CES services do reflect upon what they consider to be good care in a particular situation. It is important that such insights and action points are followed up. In our process, following up was fostered by continuously developing new CES services in close cooperation with the CEGD. Responsive evaluation research and a relationship of trust proved important vehicles for joint learning and attunement of both the traditional and new innovative CES services to the team's needs. These needs are not just the needs that are explicitly formulated by the CEGD team, they can also be needs of the CEGD according to the CES staff. We formulated 16 lessons learned during the development and execution of integrative CES services. These lessons show how integrative CES as a continuous learning process can provide a basis for new initiatives and interventions that will increase CES's relevance to care practice.

Acknowledgements We thank the team members of the CEDG for their continuing cooperation in developing CES, Katie Verest as a participating intern in the evaluation study and our colleagues at the Department of Medical Humanities, Maaike Muntinga for her help with the ethics logbook, and Yolande Voskes for setting up the initial contact with the CEDG.

Open Access This article is distributed under the terms of the Creative Commons Attribution 4.0 International License (http://creativecommons.org/licenses/by/4.0/), which permits unrestricted use, distribution, and reproduction in any medium, provided you give appropriate credit to the original author(s) and the source, provide a link to the Creative Commons license, and indicate if changes were made.

\section{References}

Abma, T. A., Baur, V. E., Molewijk, A. C., \& Widdershoven, G. A. M. (2010). Inter-ethics: Towards an interactive and interdependent bioethics. Bioethics, 24(5), 242-255. https://doi.org/10.111 1/j.1467-8519.2010.01810.x.

Abma, T. A., Molewijk, A. C., \& Widdershoven, G. A. M. (2009). Good care in ongoing dialogue. Improving the quality of care through moral deliberation and responsive evaluation. Health Care Analysis, 17(3), 217-235. https://doi.org/10.1007/s10728-008-0102-z.

Abma, T. A., \& Widdershoven, G. A. M. (2014). Dialogical ethics and responsive evaluation as a framework for patient participation. American Journal of Bioethics, 14(6), 27-29. https://doi. org/10.1080/15265161.2014.900143.

Bouman, W. P., Schwend, A. S., Motmans, J., Smiley, A., Safer, J. D., Deutsch, M. B., et al. (2017). Language and trans health. International Journal of Transgenderism, 18(1), 1-6. https://doi. org/10.1080/15532739.2016.1262127. 
Dauwerse, L., Stolper, M., Widdershoven, G. A. M., \& Molewijk, A. C. (2014). Prevalence and characteristics of moral case deliberation in Dutch health care. Medicine, Health Care and Philosophy, 17(3), 365-375. https://doi.org/10.1007/s11019-013-9537-6.

Dauwerse, L., van der Dam, S., \& Abma, T. (2012). Morality in the mundane: Specific needs for ethics support in elderly care. Nursing Ethics, 19(1), 91-103. https://doi.org/10.1177/0969733011412102.

De Snoo-Trimp, J. C., Widdershoven, G. A. M., Svantesson, H. C. W., de Vet, R., \& Molewijk, A. C. (2017). What outcomes of moral case deliberation do Dutch health care professionals perceive important and prioritize before their actual MCD participation? Bioethics, 31(4), 246-257.

Doran, E., Kerridge, I., Jordens, C., \& Newson, A. J. (2016). Clinical ethics support in contemporary health care origins, practices, and evaluation. In E. Ferlie, K. Montgomery, \& A. R. Pedersen (Eds.), The Oxford handbook of health care management (pp. 164-187). Oxford: Oxford University Press.

Finder, S. G., \& Bliton, M. J. (2011). Responsibility after the apparent end: 'Following-up' in clinical ethics consultation. Bioethics, 25(7), 413-424. https://doi.org/10.1111/j.1467-8519.2011.01910.x.

Fox, E., Bottrell, M. M., Berkowitz, K. A., Chanko, B. L., Foglia, M. B., \& Pearlman, R. A. (2010). Integrated ethics: An innovative program to improve ethics quality in health care. Innovation Journal, 15(2), 1-36.

Gender Identity Research and Education Society (GIRES). (2006). A typical gender development-A review. International Journal of Transgenderism, 9(1), 29-44.

Gerritse, K., Hartman, L. A., Antonides, M. F., Wensing-Kruger, A., de Vries, A., \& Molewijk, A. C. (2018). Moral challenges in transgender care: A thematic analysis based on a focused ethnography. Archives of Sexual Behavior, 47(8), 2319-2333.

Guba, E. G., \& Lincoln, Y. S. (1989). Fourth generation evaluation. Newbury Park: SAGE Publications.

Hartman, L. A., Widdershoven, G. A. W., \& Molewijk, A. C. (forthcoming). A plea for a more intergrative ethics support (provisional title).

Hem, M. H., Pedersen, R., Norvoll, R., \& Molewijk, A. C. (2015). Evaluating clinical ethics support in mental healthcare: A systematic literature review. Nursing Ethics, 22(4), 452-466. https://doi. org/10.1177/0969733014539783.

Hembree, W. C., Cohen-Kettenis, P. T., Gooren, L., Hannema, S. E., Meyer, W. J., Murad, M. H., et al. (2017). Endocrine treatment of gender-dysphoric/gender-incongruent persons: An endocrine society clinical practice guideline. The Journal of Clinical Endocrinology \& Metabolism, 102(11), 38693903. https://doi.org/10.1210/jc.2017-01658.

Janssens, R. M., Zadelhoff, V. E., Loo, V. G., Widdershoven, G. A. M., \& Molewijk, A. C. (2015). Evaluation and perceived results of moral case deliberation: A mixed methods study. Nursing Ethics, 22(8), 870-880. https://doi.org/10.1177/0969733014557115.

Kessels, J. (2009). Free space: Field guide to conversations. Amsterdam, Netherlands: Boom.

Lincoln, Y. S. (2003). Constructivist knowing, participatory ethics and responsive evaluation: A model for the 21 st century. In T. Kellaghan \& D. L. Stufflebeam (Eds.), International handbook of educational evaluation (pp. 69-78). Dordrecht: Springer.

McGee, G., Spanogle, J. P., Caplan, A. L., Penny, D., \& Asch, D. A. (2002). Successes and failures of hospital ethics committees: A national survey of ethics committee chairs. Cambridge Quarterly of Healthcare Ethics, 11(1), 87-93.

Molewijk, A. C., Abma, T., Stolper, M., \& Widdershoven, G. (2008). Teaching ethics in the clinic: The theory and practice of moral case deliberation. Journal of Medical Ethics, 34(2), 120-124. https:// doi.org/10.1136/jme.2006.018580.

Pedersen, R., Hurst, S. A., Schildmann, J. S. S., \& Molewijk, A. C. (2010). The development of a descriptive evaluation tool for clinical ethics case consultations. Clinical Ethics, 5(3), 136-141.

Reiter-Theil, S. (2009). Dealing with the normative dimension in clinical ethics consultation. Cambridge Quarterly of Healthcare Ethics, 18(4), 347-359. https://doi.org/10.1017/s0963180109090550.

Reiter-Theil, S. (2016). Initiating and maintaining clinical ethics support in psychiatry. Ten tasks and challenges-And how to meet them. Clinical Ethics, 11(2-3), 45-53. https://doi.org/10.1177/14777 50916649119.

Schildmann, J., Molewijk, B., Benaroyo, L., Forde, R., \& Neitzke, G. (2013). Evaluation of clinical ethics support services and its normativity. Journal of Medical Ethics, 39(11), 681-685. https://doi. org/10.1136/medethics-2012-100697.

Schildmann, J., Nadolny, S., Haltaufderheide, J., Gysels, M., Vollmann, J., \& Bausewein, C. (2017). Ethical case interventions for adult patients. Cochrane Database of Systematic Reviews, 4, 1. https://doi. org/10.1002/14651858.cd012636. 
Seekles, W., Widdershoven, G. A. M., Robben, P., van, D. G., \& Molewijk, A. C. (2016). Evaluation of moral case deliberation at the Dutch Health Care Inspectorate: A pilot study. BMC Medical Ethics, 17(1), 31. https://doi.org/10.1186/s12910-016-0114-4.

Simpson, C. (2012). Mapping our practice? Some conceptual "bumps" for us to consider. HEC Forum, 24(3), 219-226. https://doi.org/10.1007/s10730-012-9188-7.

Stolper, M. (2016). Learning by doing: Developing moral case deliberation in health care (PhD Thesis). VU University, Amsterdam.

Stolper, M., Molewijk, A. C., \& Widdershoven, G. A. M. (2015). Learning by doing. Training health care professionals to become facilitator of moral case deliberation. HEC Forum, 27(1), 47-59. https:// doi.org/10.1007/s10730-014-9251-7.

Stolper, M., Molewijk, B., \& Widdershoven, G. (2016). Bioethics education in clinical settings: Theory and practice of the dilemma method of moral case deliberation. BMC Medical Ethics, 17(1), 45. https://doi.org/10.1186/s12910-016-0125-1.

Svantesson, M., Karlsson, J., Boitte, P., Schildman, J., Dauwerse, L., Widdershoven, G. A. M., et al. (2014). Outcomes of moral case deliberation-The development of an evaluation instrument for clinical ethics support (the Euro-MCD). BMC Medical Ethics, 15(1), 30. https://doi. org/10.1186/1472-6939-15-30.

Weidema, F. C. (2014). Dialogue at work: Implementing moral case deliberation in a mental healthcare institution (PhD thesis). VU University, Amsterdam.

Weidema, F. C., van Dartel, H., \& Molewijk, B. (2016). Working towards implementing moral case deliberation in mental healthcare: Ongoing dialogue and shared ownership as strategy. Clinical Ethics, 11(2-3), 54-62. https://doi.org/10.1177/1477750916644932.

Widdershoven, G. A. M., Molewijk, A. C., \& Abma, T. A. (2009). Improving care and ethics: A plea for interactive empirical ethics. American Journal of Bioethics, 9(6-7), 99-101. https://doi. org/10.1080/15265160902893932.

Vrouwenaets, L., Hartman, L. A., Vries, A., Vries, M. C., Hein, I., \& Molewijk, A. C. (forthcoming). Dealing with moral challenges in treatment of transgender children and adolescents: evaluating the role of moral case deliberation.

ZonMw. (2019). Controversies surrounding puberty suppression in adolescents with gender identity disorder: Moving forward the ethical debate and creating an international guideline. https://www. zonmw.nl/nl/onderzoek-resultaten/kwaliteit-van-zorg/programmas/projectdetail/ethiek-en-gezon dheid-2012-2015/controversies-surrounding-puberty-suppression-in-adolescents-with-gender-ident ity-disordermoving-f/resultaten/. Accessed 11 May 2019.

Publisher's Note Springer Nature remains neutral with regard to jurisdictional claims in published maps and institutional affiliations. 